\title{
Who's Afraid of Evidence-Based Surgical Practice?
}

\author{
Arjun Rajagopalan
}

Published online: 3 February 2011

(C) Association of Surgeons of India 2011

\begin{abstract}
"Almost everyone" would be the honest answer to the question. The mantra of Evidence-based Medicine (EBM) is repeatedly chanted as a prelude to any exposition on a medical topic. But, scratch the surface, and very few of us really know what makes up good medical evidence.

The most common misperception encountered amongst doctors who would like to dip their toes into the vast ocean of EBM is that it involves a comprehensive understanding of biostatistics. This myth has to be dispelled. It is possible to analyse studies with only a working idea of a handful of definitions and terms, without delving into the mathematics that we dread, allowing the actual manipulations that are a part of biostatistical analysis to happen in a "black box". Biostatistical analysis is like a seal of approval: to be applied after a product is finished and before it is released to the world. Much like manufacturers leave the quality seal to an outside agency and concentrate on making the product to the highest specs, good medical evidence is more dependent on good experimental design than heavy-duty biostatistical analysis.

Once this myth is defused, the understanding of what makes good medical evidence is easy to grasp. In all scientific studies, there are two main sources of error: systematic and random.

Of the two sources of error in clinical studies, systematic error, or bias, is of greater concern in evaluating studies. Systematic error arises from poor study design. The primary aim of readers and critics is to detect systematic error. This does not require any knowledge outside of what we are comfortable with.
\end{abstract}

\footnotetext{
A. Rajagopalan $(\square)$

Sundaram Medical Foundation,

Chennai, India

e-mail: arjunraj@smfhospital.org
}

As a first step, we have to learn to distinguish all clinical trials as one of three varieties: observational studies, studies of diagnostic efficacy and interventional studies. Although the same tools are used in evaluation, the angle of viewing will vary depending on the basic type.

The next step, the most critical, is looking at the paper for sources of systematic error (bias). Once again, there are three possible sources. Regardless of our area of interest, all of clinical research is a mixture of three actions: observation, measurement and intervention. Each of these is a source of bias, named as sampling (observation) bias, measurement bias and comparison (interventional) bias.

As generalisations, the following broad statements can be made with regards to systematic error in studies.

1. Proper sampling is an obvious requirement. It is very rare for any study to be able to include an entire population. Adequacy of numbers has to be ensured to give the proper power to a study. This can be done in conjunction with a biostatistician, who, with the expectations that you give him, can come up with an a priori sample size determination. In general, sampling bias occurs at three points: 1 . accrual into a study from the world in general, the bias being imposed by the location and nature of the hospital or centre, 2. application of inclusion and exclusion criteria and 3. drop outs after enrolment into the trial. Purely observational studies, in particular, can be seriously flawed by sampling bias.

2. Measurement bias is a very tricky thing to detect. There are two sources: the observer and the instrument used for measurement. Using multiple observers, repeating observations by the same observer, blinding the observer to the nature of an intervention that is being measured, training observers, use of protocols and scoring systems, and comparison against known "gold standards" are some of the strategies used to keep measurement bias to the minimum. 
3. As surgeons, the majority of trials that we are likely to be interested in will be of the interventional variety. One intervention (usually something "new and improved") will be claimed to be superior to that which exists. Comparisons (controls) are the keystone of these trials and it is therefore imperative that the comparisons be fair: apples to apples and not apples to oranges. Pitching the assessed intervention against a proper, matched control is of the essence in interventional studies. Controls may be obtained by retrospective accrual of patients and data, case-controlled matching, or in a randomised, prospective trial. At the present time, it is universally accepted that the randomised controlled trial (RCT) is the only way we can ensure minimising comparison bias. The ethics and responsibilities of random allocation are onerous, even repugnant to some, yet, the only path to the truth for now. Retrospective analysis of case series, however large, is not acceptable evidence. Case reports are merely snapshots of the strange and wonderful things that this world abounds in and are not accepted in high quality clinical journals any more.

The critical step in EBM is the detection of the degree of systematic error. A poorly designed study can never be set right by rigorous statistical analysis.

Random error arises from the nature of distribution of measured values in any biological sample. We are all familiar with the bell-shaped curve in which values will be dispersed when measured in any normal population. In the simplest sense, all scientific studies attempt to establish differences. When differences occur in studies, it is possible that this variability might merely be due to the natural distribution of values and not the effect of any specific event or intervention. The aim of biostatistical analysis is to determine whether demonstrated differences have occurred by chance (random) or whether this is a real, repeatable difference that furthers our knowledge and can be used to our advantage. This exercise is best left to trained biostatisticians. Their results, regardless of the complexity and arcane nature of the tests used, will be expressed as certain concepts and measures of confidence, all of which are easy to understand and interpret with minimal effort. The list includes: ' $p$ ' values and confidence intervals; odds and risk ratios; measures of correlation and regression; kappa values, likelihood ratios (assessment of sensitivity and specificity); and estimation of numbers needed to treat (NNT). Armed with this small but effective range of implements, we can confidently handle the biostatistical monster that we have been terrorised by all these years.

If both elements - systematic and random error-are found acceptable, then the study is given the stamp of being good evidence.

Evidence-based medical practice is the application of evidence in Medicine in a four step fashion:

1. That which is true and proven true should be incorporated into practice or continued if already in use - a "standard of care".

2. That which may be true but not yet proven so must be submitted to well done research before being included as a "standard of care".

3. That which is proven false must be avoided or eliminated from existing practice.

4. That which is not likely to be true and is unproven is a source of great danger and must be avoided at all costs. This segment is fertile ground for quack remedies and fantastic claims.

As surgeons, we pride ourselves on our stamina, commitment and the ability to do difficult tasks well. The practice of evidence-based surgery is a responsibility that we cannot dismiss as fit only for ivory-towered academicians. Too much of yesterday's dicta have crumbled when examined under the lens of EBM. A surgeon's skill is largely an ability to dissect well. It is acquired over years of practice. Likewise, evidence-based surgical practice is dependent on the ability to dissect scientific studies with skill. 